\title{
Los recursos hídricos de Florida ${ }^{1}$
}

\author{
Tatiana Borisova, Tara Wade ${ }^{2}$
}

\section{Introducción: ¿Por qué son importantes los recursos hídricos?}

"El agua es la sangre de nuestros cuerpos, nuestra economía, nuestra nación y nuestro bienestar." (Stephen Lee Johnson, Director de la EPA bajo la administración de G.W. Bush).

Esta cita resume la importancia de los recursos hídricos. Utilizamos agua para beber, jardinería y otros usos domésticos, en agricultura (por ejemplo, el riego), y en producción de energía y procesos industriales (por ejemplo, para enfriamiento en generación de energía termoeléctrica). Los recursos hídricos limpios y abundantes también son importantes para nuestras actividades recreativas (por ejemplo, paseos en bote, natación o pesca). El agua también sostiene la vida silvestre (como los manatíes) y es parte integral del medio ambiente de la Florida (Figura 1).

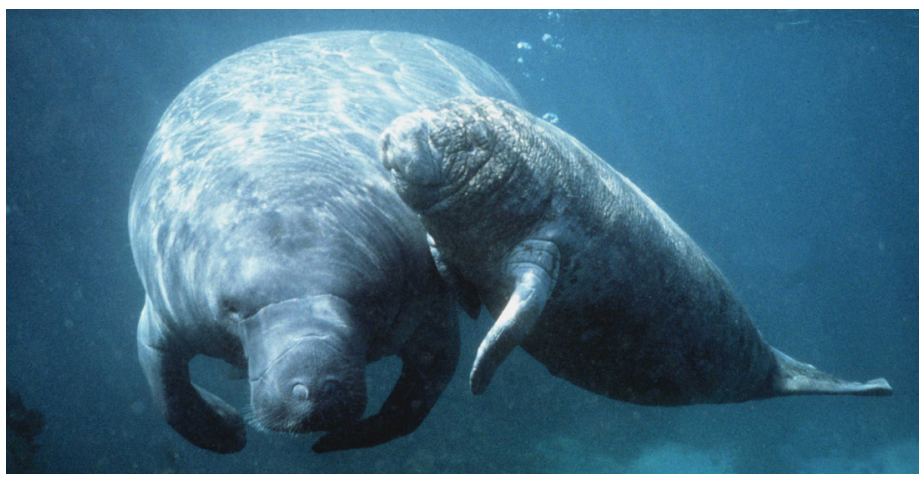

Figura 1. En noviembre, los manatíes migran a las aguas costeras más cálidas, como Crystal River en la costa occidental de la Florida.

Créditos: UF/IFAS File Photo
El uso del agua está aumentando junto con la población de la Florida. Los floridanos dependen de las reservas subterráneas de agua dulce, llamadas acuíferos, para abastecer las diversas necesidades de agua (USGS 2016a). En algunas regiones de la Florida, esta reserva de agua dulce subterránea ya no puede sostener las crecientes demandas de agua de la población, y al mismo tiempo alimentar los ríos, manantiales y lagos de la Florida. Con sequías periódicas, puede producirse escasez de agua dulce. La sequía y la escasez de agua en el estado han hecho que los planificadores urbanos y los encargados de formular políticas presten más atención al uso del agua, al desarrollo del suministro de agua y al manejo de los recursos hídricos. Las encuestas de opinión pública de la Florida demuestran que la mayoría de los floridanos clasifican el agua como una cuestión de suma importancia para el estado (UF / IFAS Centro de Estudios Públicos 2016). Una mejor comprensión de los recursos hídricos de la Florida es un primer paso hacia la optimización del uso actual del agua dulce y puede asegurar los recursos hídricos adecuados en el futuro.

\section{El Ciclo Hidrológico: De donde viene el agua y a dónde va}

Toni Morrison, una novelista estadounidense, dijo una vez que "toda el agua tiene una memoria perfecta y siempre trata de volver a donde estaba." De hecho, el agua se mueve constantemente. El ciclo hidrológico (o de agua) es la circulación/distribución continua del agua en la superficie de la tierra, en el suelo y en la atmósfera (USGS 2016b). El

1. This document is FE1044, one of a series of the Food and Resource Economics Department, UF/IFAS Extension. Original publication date October 2018. Visit the EDIS website at https://edis.ifas.ufl.edu for the currently supported version of this publication.

2. Tatiana Borisova es profesora asociada del Departamento de Economía de Alimentos y Recursos; y Tara Wade, profesora asistente, Departamento de Economía de Alimentos y Recursos, Centro de Investigación y Educación del Suroeste de Florida, Extensión UF/IFAS, Immokalee, FL.

The Institute of Food and Agricultural Sciences (IFAS) is an Equal Opportunity Institution authorized to provide research, educational information and other services only to individuals and institutions that function with non-discrimination with respect to race, creed, color, religion, age, disability, sex, sexual orientation, marital status, national origin, political opinions or affiliations. For more information on obtaining other UF/IFAS Extension publications, contact your county's UF/IFAS Extension office. 
origen y la disponibilidad del agua son fundamentales para entender cómo el agua "hace ciclos" a través del medio ambiente. Hay cinco procesos básicos en el ciclo hidrológico: (1) condensación, (2) precipitación, (3) infiltración, (4) escorrentía, y (5) evapotranspiración (Figura 2). Estos procesos pueden ocurrir simultáneamente y, a excepción de la precipitación, continuamente. Discutiremos estos cinco procesos en el contexto de la Florida.

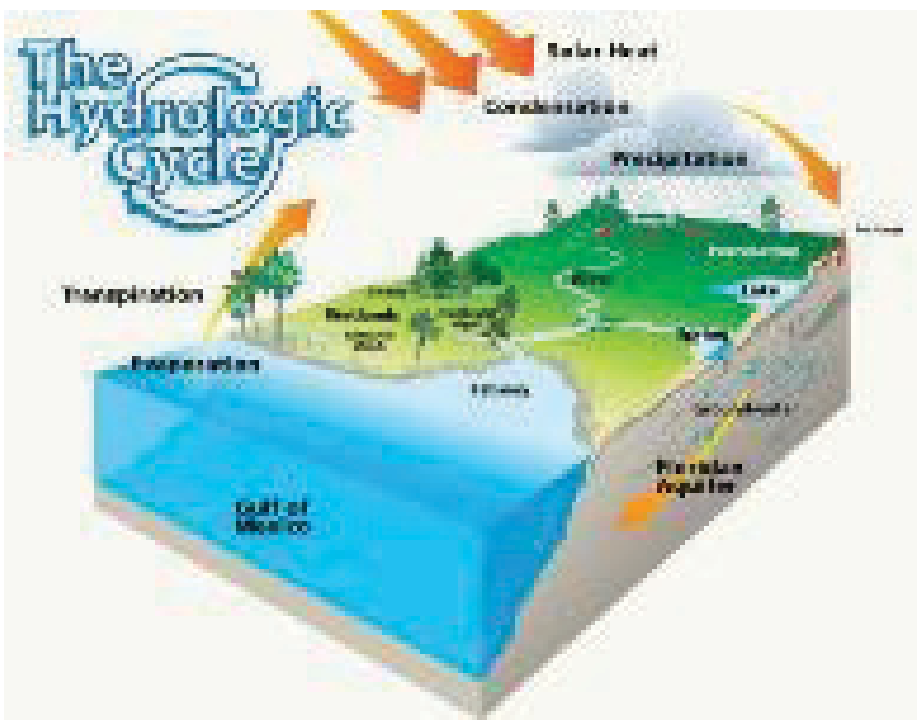

Figura 2. Ciclo hidrológico.

Créditos: SWFWMD

\section{Condensación}

Este proceso es familiar a cualquier floridano que deja su coche afuera en la noche. Por la mañana, las ventanas del coche están cubiertas con pequeñas gotas de agua o rocío. En general, la condensación es el proceso por el cual el vapor de agua cambia de fase gaseosa a líquida. Esto ocurre cuando el aire húmedo se enfría. Mientras se enfría, el vapor de agua forma gotitas diminutas que se adhieren al polvo, sal y partículas de humo en el aire y luego forman rocío o niebla. La formación de nubes es otro ejemplo del proceso de condensación que ocurre en altitudes más altas donde el aire es más frío.

\section{Precipitación}

La precipitación es el producto de la condensación del vapor de agua atmosférico. La lluvia es un ejemplo de precipitación, otra etapa del ciclo hidrológico. La precipitación se produce cuando las gotas de agua formadas en altitudes más altas aumentan de tamaño y aumentan de peso, causando que las gotas caigan debido a la gravedad. Dependiendo de las condiciones, la precipitación ocurre más típicamente como lluvia, granizo, nieve o aguanieve.

Florida tiene un clima subtropical/tropical y recibe un promedio de 55 pulgadas de lluvia por año (promedios estatales para 1981-2010) (OSU/PRISM 2014, Centro Climático de Florida 2017). Esto es casi dos veces el promedio nacional de 31 pulgadas por año, y cinco veces más alto que el nivel de lluvia en el estado más seco de los Estados Unidos, Nevada (11 pulgadas por año, promedios para 1981-2010) (NOAA/NCEI 2014, 2017).

Las precipitaciones varían en cantidades y en intensidad de una región de la Florida a otra, con la mayor precipitación anual en el noroeste de la Florida (el "Panhandle") y en las zonas costeras del sureste de Florida (Figura 3). En estas regiones, la precipitación media es de aproximadamente 60 pulgadas por año. Otras regiones reciben menos lluvia. Por ejemplo, los Cayos de la Florida tienen un promedio de 40 pulgadas de lluvia anualmente (promedio para 1981-2010) (OSU/PRISM 2014; Centro Climático de Florida 2017a).

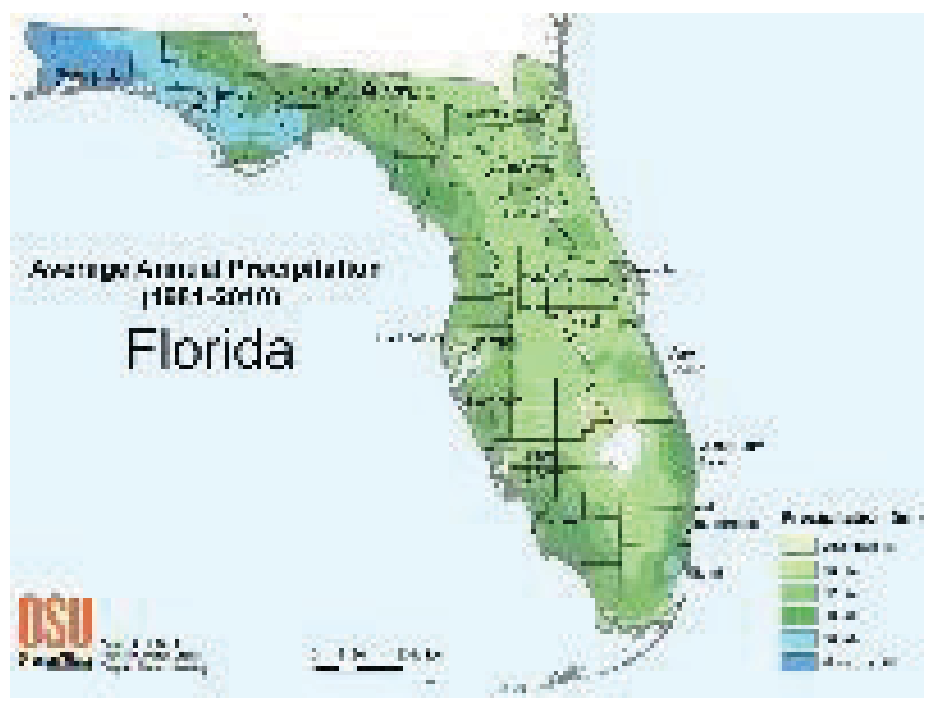

Figura 3. Precipitación anual promedio de la Florida, 1981-2010. Créditos: OSU/PRISM

Las variaciones estacionales en las precipitaciones también son evidentes. Tradicionalmente, el verano es la estación más húmeda en Florida, con más de la mitad de la lluvia anual que ocurre durante la "temporada lluviosa" de junio a septiembre (Figura 4). Sin embargo, este patrón de precipitación estacional varía (vea los valores mínimos y máximos mensuales de precipitación en la Figura 4). Durante la estación húmeda, las tormentas tropicales son normales en la Florida, con algunas entregando más de 10 pulgadas de lluvia durante un período de 24 horas (causando inundaciones). El huracán Easy (en 1950) tiene la precipitación más alta estimada de casi 39 pulgadas en 24 horas en Yankeetown (centro-oeste de la Florida). El huracán Jeanne (en 1980) tiene la mayor precipitación medida oficialmente del estado de aproximadamente 23 pulgadas dentro de un período de 24 horas (Centro Climático de la Florida 2017b). Por el contrario, la primavera de 2017 será recordada por sus condiciones de sequía generalizada, donde 
en mayo, un asombroso 66\% del estado registró sequía de varios niveles de gravedad debido a la baja precipitación en los primeros meses del año (Rice 2017).

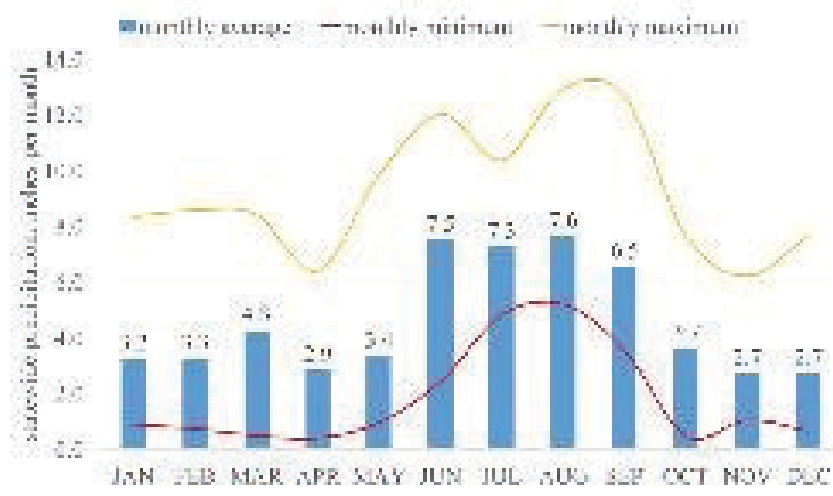

Figura 4. Precipitación mensual a nivel estatal, 1981-2010. Créditos: Centro Climático de la Florida

En la Florida, se usan volúmenes significativos de agua para el riego residencial y agrícola. En los meses secos de primavera, los propietarios residenciales dependen del riego suplementario para el paisajismo de sus patios. A su vez, muchos cultivos agrícolas en la Florida se plantan en el otoño cuando la temperatura del aire se enfría, con la cosecha en las estaciones de invierno y primavera, utilizando el riego durante los meses secos de invierno y primavera para el riego suplementario y la protección contra heladas. El aumento de la eficiencia del uso del agua es una alta prioridad para los floridanos. Para sugerencias relacionadas con las prácticas de riego residencial, vea la información de Florida Friendly Landscaping ${ }^{\mathrm{Tm}}$ en el sitio web de la Universidad de Florida (http://fyn.ifas.ufl.edu/homeowners/nine_principles.htm). Para las mejores prácticas de gestión (BMP) relacionadas con el riego agrícola, consulte los manuales de BMP disponibles en el sitio web del Departamento de Agricultura y Servicios al Consumidor de la Florida (http:// www.freshfromflorida.com/Business-Services/Water/ Agricultural-Best-Management-Practices).

Capturar y almacenar lluvia en embalses para reducir la necesidad de bombear agua de los acuíferos durante los meses más secos, es también una estrategia que está siendo explorada por los municipios de la Florida.

\section{Infiltración, percolación y recargas}

Cuando la lluvia llega a la superficie de la Tierra, puede entrar en el suelo (infiltración), recogerse en arroyos y lagos superficiales (escorrentía), o regresar a la atmósfera como vapor de agua (evapotranspiración). Las fases de la escorrentía y la infiltración están altamente interrelacionadas, y están influenciadas por la forma de precipitación, el tipo y la cantidad de cobertura vegetal, la topografía y la permeabilidad del suelo.

La infiltración ocurre cuando el agua entra por primera vez en la zona de la superficie del suelo. El agua subterránea se recoge cuando el agua que no es utilizada por las plantas se infiltra (o se filtra) hacia abajo hasta que encuentra una zona (estrato) donde los poros en el suelo o las rocas están saturados. Las capas subterráneas de material poroso que están saturadas con agua se llaman acuíferos. El nivel del agua puede subir y bajar en acuíferos superficiales, dependiendo de las condiciones locales de lluvia. Cuando un acuífero de aguas subterráneas poco profundas está subyacente a un estrato de baja permeabilidad denominado unidad de confinamiento (por ejemplo, arcilla) (Figura 5), el agua debe moverse lateralmente a través del acuífero y emerger en una superficie de manantial, arroyo o lago. Por el contrario, cuando los niveles del agua subterránea son bajos, el agua puede fluir en la dirección opuesta, desde arroyos superficiales y lagos hasta el acuífero poco profundo. A veces, el agua dulce existe a gran profundidad en acuíferos confinados, donde el acuífero está confinado debajo de un estrato de baja permeabilidad (por ejemplo, cavidades bajo la capa de arcilla) (Figura 5). Un acuífero confinado a veces puede mantener el agua bajo suficiente presión, permitiendo que el agua se eleve por encima de la capa de confinamiento cuando un pozo fuertemente encajado penetra en la unidad de confinamiento (piense en cómo funciona una fuente de agua). Estos son conocidos como acuíferos artesianos. Cuando son aprovechados, a veces producen pozos artesianos de flujo libre. Los manantiales de origen natural también resultan de este mismo fenómeno (Figura 5).

El agua también puede entrar en acuíferos a través de zonas de recarga donde la capa freática emerge en la superficie o donde la capa de confinamiento se rompe por fallas y fisuras naturales que permiten la infiltración descendente del agua (Figura 5). Tenga en cuenta que las zonas de recarga pueden estar a cierta distancia de los manantiales o pozos que son alimentados por el acuífero.

\section{Los acuíferos de la Florida}

Florida tiene varios acuíferos prolíficos (Figura 6) que producen grandes cantidades de agua para los pozos, arroyos, lagos y manantiales (algunos de los más grandes del mundo). La principal fuente de agua subterránea para la mayor parte del estado es el Acuífero Floridano-la fuente del suministro de agua municipal en el norte y el centro de la Florida. También rinde agua a miles de pozos domésticos, industriales y de riego en todo el estado. 


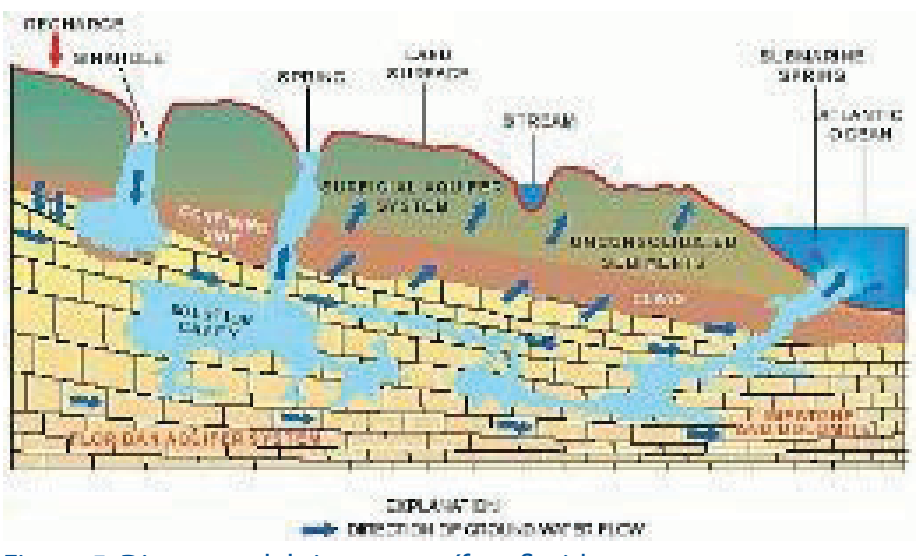

Figura 5. Diagrama del sistema acuífero floridano. Créditos: USGS

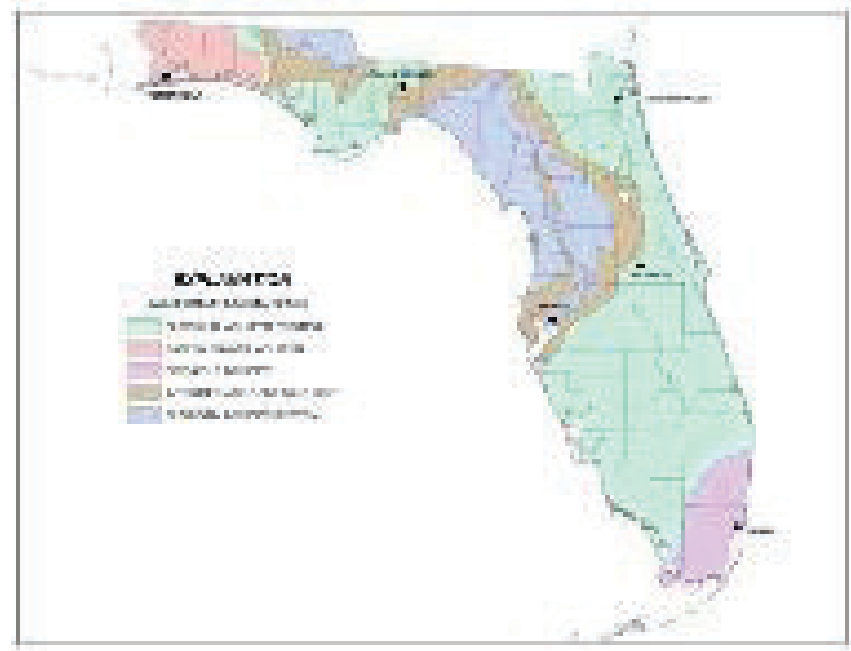

Figura 6. Los acuíferos de la Florida.

Créditos: FDEP

Un acuífero superficial no-artesiano está presente en gran parte del estado, pero no es una fuente importante de agua subterránea en la mayoría de las áreas debido a que hay un mejor suministro disponible de los acuíferos más profundos. Sin embargo, en las zonas rurales donde las necesidades residenciales de agua son relativamente más pequeñas en comparación con otras áreas, este acuífero es aprovechado por pozos de pequeño diámetro. El agua en este acuífero poco profundo se deriva principalmente de la lluvia local.

Un acuífero no-artesiano de arena y grava es la principal fuente de agua subterránea en la parte occidental extrema del "Panhandle" de la Florida (la región noroeste). El agua en el acuífero de arena y grava se deriva principalmente de las precipitaciones locales y es de buena calidad química. Los pozos que extraen este acuífero proporcionan la mayor parte del agua subterránea utilizada en los condados de Escambia y Santa Rosa, y parte del condado de Okaloosa.
El acuífero no artesiano de Biscayne subyace en un área de aproximadamente 3.000 millas cuadradas en los condados de Miami-Dade, Broward y Palm Beach, en la costa oriental de Florida. El agua en el acuífero Biscayne se deriva principalmente de las lluvias locales $y$, durante los periodos secos, de los canales vinculados al lago Okeechobee. El acuífero Biscayne es un importante suministro de agua para las ciudades de la costa oriental de Florida.

Las capas gruesas de piedra caliza porosa del acuífero floridano subyacen a todo el estado y se extienden más allá de las fronteras estatales, hasta Georgia, Carolina del Sur, Alabama y Mississippi. En el sur de la Florida, el agua del acuífero floridano está muy mineralizada (es decir, salada) para ser utilizable. El agua en el acuífero floridano se reabastece con lluvias en el norte y centro de la Florida (así como en el sur de Georgia y el sur de Alabama), donde el acuífero emerge en la superficie o está cubierto por materiales permeables o donde el material confinante está perforado por sumideros. En estas áreas, es especialmente importante observar prácticas apropiadas cuando se trabaja en jardinería, manejo de sistemas sépticos o cultivos agrícolas. Estas prácticas pueden afectar directamente la calidad del agua en el acuífero y, por lo tanto, la calidad del agua retirada para beber y otros propósitos y la calidad del agua en los manantiales alimentados por el sistema acuífero.

Para más información sobre los acuíferos de Florida, véase FDEP (2015). Puede obtener más información sobre el sistema acuífero de la Florida viendo videos recopilados en el sitio web del Servicio Geológico de los Estados Unidos (https://fl.water.usgs.gov/floridan/visual_gallery.html).

\section{Los manantiales de la Florida}

Con excepción de aquellas áreas donde sus formaciones calcáreas rompen la superficie del suelo, el acuífero floridano subyace varios cientos de pies de sedimento, incluyendo gruesos lechos de material relativamente impermeable que restringen el movimiento ascendente del agua (Marella y Berndt 2005). Esta restricción hace que el acuífero tenga presión artesiana y haga que el agua se mueva a través de las aberturas en capas impermeables que crean manantiales (Figura 7). En Florida, hay más de 1.000 manantiales, incluyendo más de 30 manantiales de primera magnitud con un flujo promedio de más de 100 pies cúbicos por segundo (64.6 millones de galones por día) (Knight 2017). El agua de manantial emerge de las cavidades en la caliza porosa del acuífero floridano, y a menudo contribuye al flujo o nivel del agua en arroyos y lagos. Los manantiales dependen de los mismos recursos de los cuales retiramos agua para los 
suministros públicos de agua, pozos de agua privados y producción agrícola. Los aumentos en las extracciones de agua para cualquier propósito también reducen los flujos de manantiales. El proverbio chino "cuando usted bebe agua, recuerda el manantial" se aplica a la Florida literalmente.

\section{La intrusión de agua salada}

La geografía de la Florida como una península entre dos cuerpos de agua salada crea el potencial para la intrusión de agua salada en los acuíferos (es decir, en el suministro de agua dulce subterránea). El agua salada es más densa que el agua dulce y ejerce una presión constante para permear los acuíferos porosos. Mientras los niveles de agua dulce en los acuíferos estén más altos que el nivel del mar, la presión de agua dulce evita que el agua salada se mueva hacia el interior y hacia arriba en los acuíferos. Por ejemplo, el nivel de agua que fluye a través de los canales costeros del sur de Florida es generalmente varios pies sobre el nivel del mar, que es suficiente para evitar que el agua del océano se mueva hacia el interior y hacia arriba en el acuífero. Sin embargo, si durante los periodos secos, los niveles de agua dulce en los canales sin esclusas y represas caen o están por debajo del nivel del mar, el agua salada se movería hacia arriba en los canales.

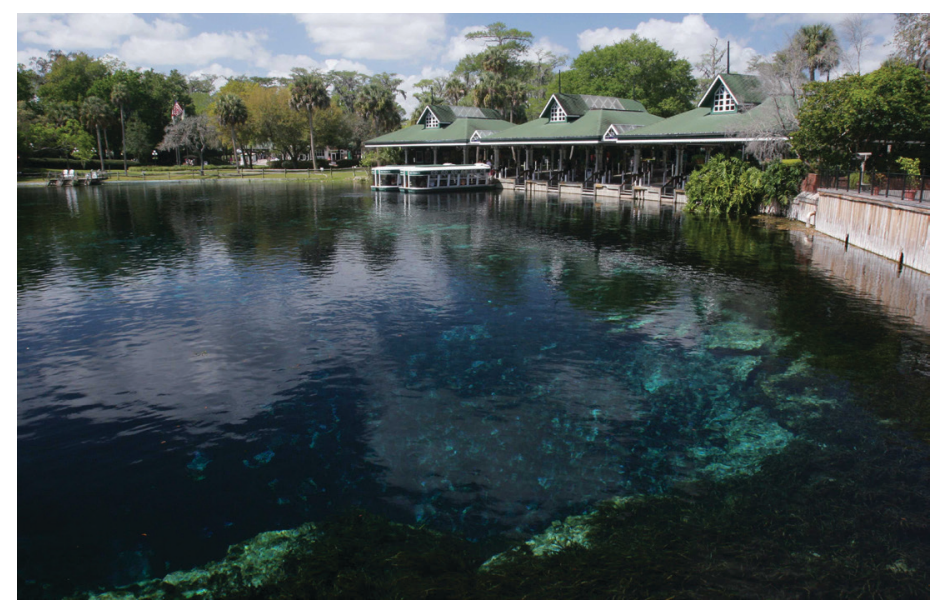

Figura 7. Silver Springs, Florida.

Créditos: UF/IFAS File Photo

En algunos lugares, el bombeo excesivo de un pozo puede aumentar la intrusión de agua salada. Si el agua se bombea a una velocidad más rápida que el acuífero se repone, la presión del agua dulce sobre el agua salada en la masa terrestre disminuye. Esta disminución puede hacer que el nivel de la interfaz agua salada-agua dulce aumente en el acuífero, degradando la calidad del agua. Este problema debe ser controlado por una cuidadosa atención a la ubicación del pozo y las tasas de bombeo. El problema de la intrusión de agua salada se ve exacerbado por períodos de sequía cuando no hay suficientes precipitaciones para reponer los acuíferos de agua dulce.
El Río Apalachicola, que fluye hacia el sur desde su cabecera al norte de Atlanta, Georgia, después de la confluencia de los Ríos Flint y Chattahoochee en la frontera Georgia-Florida, es el caudal más grande de las corrientes de agua de Florida por volumen de agua. El Río Apalachicola drena 17.200 millas cuadradas en Alabama y Georgia, y 2.400 millas cuadradas en la Florida. Entre 1978 y 2012, la descarga media del Río Apalachicola en Sumatra, Florida (el punto medio de la longitud del río en Florida) fue de 24.000 pies cúbicos por segundo (o 15.000 millones de galones por día), con una variación entre aproximadamente 10.000 y 37.000 pies cúbicos por segundo (o de 6 mil millones a 24 mil millones galones por día) (USGS 2014a). A pesar de que el río lleva volúmenes significativos de agua, la necesidad de agua es cada vez mayor debido al crecimiento de la población en los tres estados. Desde la década de 1990, los tres estados han discutido sobre la cantidad de agua que cada uno de los estados debe recibir del sistema fluvial (prioridades: Georgia, uso público de agua en Atlanta; Alabama, riego agrícola; y Florida, producción de ostras de agua dulce en la bahía Apalachicola). En otras palabras, una gestión y asignación inteligente de agua es muy importante.

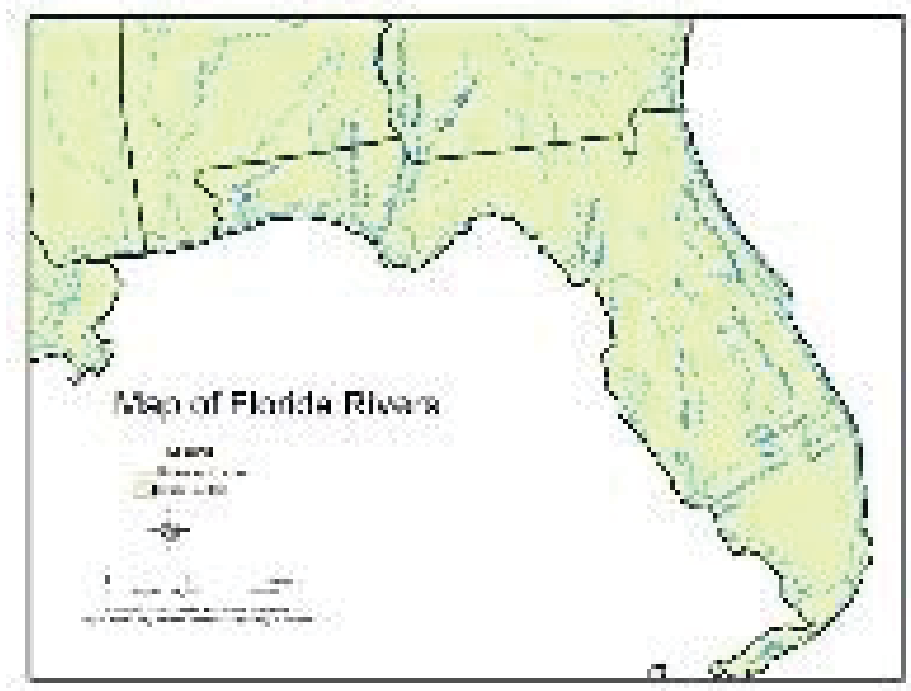

Figura 8. Los ríos de la Florida.

Créditos: USGS

El Río Suwannee (el segundo río más grande de la Florida por el volumen de descarga) drena cerca de 11.000 millas cuadradas desde sus cabeceras en la marisma de Okefenokee en el sur de Georgia hasta su boca en el golfo de México. En la estación de medición en Wilcox, Florida (33 millas al norte del Golfo de México), el Río Suwannee descarga alrededor de 10.000 pies cúbicos por segundo (6.000 millones de galones por día, promedio para 1930-2013). La variación es entre 3.000 y 25.000 pies cúbicos por segundo (o entre 2 mil millones y 16 mil millones galones por día) 
(USGS 2014b). El Río Santa Fe fluye hacia el interior del río Suwannee, al igual que una serie de manantiales, como Troy, Ichetucknee, Fanning y Manatee.

El Río Choctawhatchee (el tercer río más grande de la Florida por el volumen de descarga) drena 3.100 millas cuadradas en el sureste de Alabama y 1.500 millas cuadradas en el noroeste de la Florida (el Panhandle). La bahía Choctawhatchee se abre al Golfo de México cerca de Fort Walton Beach y Niceville. En la estación de medición cerca de Bruce, Florida, unas 21 millas de la boca del río Choctawhatchee, la descarga media es de unos 7.000 pies cúbicos por segundo (más de 4 mil millones de galones por día, promedio para 1931-2013). La variación es entre 3.000 y 12.000 pies cúbicos por segundo (o entre 2 mil millones y 8 mil millones galones por día) (USGS 2014c).

El Río Escambia y sus afluentes drenan 3,760 millas cuadradas en Alabama y 425 millas cuadradas en el noroeste de la Florida antes de fluir hacia la bahía de Pensacola a una tasa de casi 7.000 pies cúbicos por segundo (más de 4 mil millones de galones por día, medida cerca de Molino, Florida, 1988-2013) (USGS 2014d).

De los cinco ríos más grandes de Florida, sólo el Río St. Johns está completamente dentro de las fronteras del estado. Para controlar el flujo o la calidad del agua en los otros cuatro ríos más grandes de la Florida es necesario coordinar esfuerzos con otros estados. El Río St. Johns drena cerca de 9.400 millas cuadradas de marismas ubicadas al oeste de Vero Beach hasta su boca en el Océano Atlántico en Jacksonville. Es uno de los pocos ríos de los Estados Unidos que fluye hacia el norte. En su boca cerca de Jacksonville, el flujo es de unos 7.000 pies cúbicos por segundo (más de 4 mil millones de galones por día, un promedio para 1970-2011) (USGS 2014e). El Río St. Johns conecta siete lagos importantes, desde el lago Washington hasta el lago George. Su afluente, el río Ocklawaha, conecta nueve lagos, desde el lago Apopka hasta el lago Lochloosa.

Otros arroyos importantes incluyen el Río Kissimmee (con las cabeceras cerca de Orlando, que fluye al sur y que vacía en el lago Okeechobee en el centro del estado); el Río Peace (fluyendo a Charlotte Harbor), y el Río Withlacoochee (fluyendo al noroeste desde Green Swamp en el centro de la Florida y vaciando en el golfo de México cerca de Yankeetown). Además, el Canal de St. Lucie conecta el lago Okeechobee al Océano Atlántico en la costa oriental cerca de Stuart, y el Canal de Caloosahatchee conecta el lago Okeechobee al golfo de México en la costa occidental cerca de Fort Myers. Juntos, estos dos canales forman una vía fluvial navegable que atraviesa el estado. Otros canales del lago Okeechobee al Océano Atlántico son los canales de Hillsboro, North New River, Miami, y West Palm Beach (Fernald y Purdum 1998).

Además de los ríos, Florida tiene más de 7.700 lagos, siendo el lago más grande, el lago Okeechobee, el que está entre los 10 lagos más grandes de las áreas superficiales de los Estados Unidos (Fernald y Purdum 1998; FDEP 2016). Florida también tiene muchos tipos de humedales, incluyendo los Everglades (el sur de Florida) y Green Swamp (Florida central), que proporcionan hábitats para una variedad de plantas y vida silvestre, y sirven como áreas de recarga de agua subterránea.

En resumen, los arroyos, ríos, manantiales, lagos y humedales producidos por la fase de escorrentía del ciclo hidrológico de la Florida son familiares a los floridanos como fuentes de abastecimiento de agua, atracciones recreativas, rutas de transporte y refugios para las abundantes poblaciones de peces y vida silvestre del estado.

\section{Escorrentía}

La escorrentía es el agua que no entra al suelo, sino que se acumula en ríos, arroyos, canales o lagos. Esta agua se evapora, se infiltra en el suelo o fluye hacia el océano. A su vez, el agua subterránea puede fluir cerca de la superficie y luego descargarla para alimentar manantiales, arroyos, ríos o lagos. Tenga en cuenta que la escorrentía superficial, así como las tasas de infiltración y descarga del agua subterránea, dependen del uso de la tierra, los suelos y las condiciones meteorológicas. Con el crecimiento de la población y los cambios relacionados con el uso de la tierra, alteramos significativamente la relación entre el volumen de lluvia que sale de la tierra y el volumen que recarga los acuíferos. Los aumentos en el área cubierta con superficies impermeables, tales como carreteras, entradas de vehículos, y casas, pueden disminuir la tasa de recarga del acuífero mientras se aumenta el volumen de escorrentía. El aumento de los volúmenes de escorrentía puede afectar la calidad del agua en los ríos, lagos y canales, ya que la escorrentía puede transportar una variedad de contaminantes. De hecho, como Alan Levere, Departamento para la Protección del Medio Ambiente de Connecticut, declaró: "Un río es la boleta de calificaciones para la cuenca."

En Florida, la escorrentía superficial y la descarga de agua subterránea alimentan más de 1.700 arroyos y ríos (FDEP 2016). De los cinco ríos más grandes de la Florida, cuatro están en las cuencas de drenaje del norte de la Florida: Apalachicola, Suwannee, Choctawhatchee y Escambia (Figura 8). El quinto arroyo más grande es el Río St. Johns, 
que fluye hacia el norte desde sus cabeceras cerca de Vero Beach a su boca en el Océano Atlántico en Jacksonville.

\section{Evapotranspiración}

Una etapa adicional del ciclo hidrológico es la evapotranspiración. La evapotranspiración es un proceso combinado de evaporación de las superficies y transpiración a través de las hojas de las plantas (Irmak y Haman 2014).

Generalmente, la evaporación es el proceso por el cual el agua se convierte a su forma gaseosa (vapor de agua). Parte de la precipitación se evapora de la superficie terrestre de nuevo a la atmósfera. El potencial de evaporación de un área depende de las condiciones atmosféricas tales como la temperatura y la velocidad del viento. La evaporación también se ve afectada por factores como la permeabilidad del suelo, el tipo y la cantidad de cobertura vegetal y la pendiente del terreno. Por ejemplo, la evaporación es relativamente baja en partes del noroeste de la Florida. Esta área está bien drenada y, en comparación con otras partes de la Florida, tiene pendientes pronunciadas. Gran parte del área está cubierta por suelos permeables que pasan fácilmente las precipitaciones a un acuífero poco profundo. Una capa impermeable del suelo subyacente en el acuífero poco profundo en esta área asegura que la mayor parte de la lluvia aparezca en los arroyos. Por el contrario, para las porciones del extremo sur de la Florida, donde la topografía es plana y el drenaje es pobre, el agua está fácilmente disponible para la evaporación.

A su vez, la transpiración es el proceso por el cual la humedad en las plantas es devuelta a la atmósfera a través de las hojas de la planta. Muchas plantas dependen de la precipitación que infiltra el suelo desde la superficie.

Hay una deficiencia de agua cuando la evapotranspiración potencial (es decir, la evaporación más la demanda de humedad por las plantas) excede la evapotranspiración real (es decir, la humedad del suelo que está realmente disponible para la evaporación y para las plantas). Los presupuestos mensuales de agua climática indican que, en Key West, la deficiencia de agua persiste durante todo el año; en la región noroeste (el "Panhandle"), las deficiencias de agua rara vez ocurren; y en el resto del estado, las deficiencias de agua son comunes en invierno y primavera.

\section{Conclusiones}

El ciclo hidrológico del agua es una manera útil de describir y organizar los recursos hídricos de la Florida. El costo y la factibilidad de hacer los suministros de agua disponibles para usos municipales, agrícolas e industriales está determinado en gran medida por los patrones del ciclo de lluvia, escorrentía e infiltración a través del tiempo y el espacio.

Es importante recordar que mientras la Florida recibe lluvias significativas cada año, la mayor parte de esta agua vuelve al medio ambiente mediante la evapotranspiración y salida de los ríos. Sólo un porcentaje muy pequeño se infiltra en el suelo para reponer los depósitos subterráneos de agua dulce/acuíferos de los que todos dependemos para el agua potable. Todos deben ser conscientes de que el aumento de las extracciones de agua de los acuíferos para las necesidades humanas reduce la cantidad de agua disponible para alimentar los manantiales, ríos y humedales no sólo ahora, sino también en los próximos años.

\section{Fuentes}

Fernald E.A., y E.D. Purdum (editores). 1998. Water Atlas of Florida. Tallahassee, FL: FSU/ISPA.

Centro Climático de la Florida. 2017a. Precipitation. Tallahassee, FL: Florida Climate Center. http://climatecenter.fsu.edu/products-services/data/statewide-averages/ precipitation

Centro Climático de la Florida. 2017b. Hurricanes. Tallahassee, FL: Florida Climate Center. http://climatecenter. fsu.edu/topics/hurricanes

Departamento de Protección Ambiental de la Florida (FDEP). 2015. Aquifers. Tallahassee, FL: FDEP. http://www. dep.state.fl.us/swapp/aquifer.asp\#

Departamento de Protección Ambiental de la Florida (FDEP). 2016. Final Integrated Water Quality Assessment for Florida: 2016 Section 303(d), 305(b), and 314 Report and Listing Update. Tallahassee, FL: FDEP.

Irmak S., y D.Z. Haman. 2014. Evapotranspiration: Potential or Reference? EDIS \#AE256. Gainesville, FL: UF/IFAS Extension. http://edis.ifas.ufl.edu/ae256

Knight, R. 2017. Florida: Land of 1,000 Springs. High Springs, FL: Florida Springs Institute. http://floridaspringsinstitute.org/springs-of-florida

Marella, R.L., y M.P. Berndt. 2005. Water Withdrawals and Trends from the Floridan Aquifer System in the Southeastern United States, 1950-2000. Reston, VA: US Geological Survey (USGS). 
Asociación Nacional Oceánica y Atmosférica, Centros Nacionales de Información Medioambiental (NOAA/ NCEI). 2017. National Centers for Environmental Information, Climate at a Glance: U.S. Time Series. Asheville, NC: NOAA/NCEI. http://www.ncdc.noaa.gov/cag/

Asociación Nacional Oceánica y Atmosférica, Centros Nacionales de Información Medioambiental (NOAA/ NCEI). 2014. Extremes in U.S. Climate. Asheville, NC: NOAA/NCEI. http://www.ncdc.noaa.gov/extremes/ extreme-us-climates.php

OSU/PRISM. 2014. Florida Average Annual Precipitation, 1981-2010. Corvalis, OR: PRISM Climate Group, Oregon State University.

Rice, D. 2017. Move over, California: Florida has the USA's worst drought. USA Today, May 5. https://www.usatoday. com/story/weather/2017/05/05/move-over-californiaflorida-has-usas-worst-drought/101331462/

Servicio Geológico de los Estados Unidos (USGS). 2016 a. Water-Use in Florida. Reston, VA: USGS. https://fl.water. usgs.gov/infodata/wateruse.html

Servicio Geológico de los Estados Unidos (USGS). 2016b. The Water Cycle - USGS Water Science School: Springs - The Water Cycle. Reston, VA: USGS. https://water.usgs.gov/edu/ watercyclesprings.html

Servicio Geológico de los Estados Unidos (USGS). 2014a. Surface-Water Annual Statistics for Florida. USGS 02359170 Apalachicola River near Sumatra, Florida. Reston, VA: USGS. http://waterdata.usgs.gov/ fl/nwis/annual/?referred_module $=$ sw\&site_no $=023591$ 70\&por_02359170_1=2396790,00060,1,1978,2014\&ye ar_type $=$ C\&format $=$ html_table\&date_format $=$ YYYY-MMDD\&rdb_compression=file\&submitted_form =parameter_ selection_list

Servicio Geológico de los Estados Unidos (USGS). 2014b. Surface-Water Annual Statistics for Florida. USGS 02323500 Suwannee River near Wilcox, Florida. Reston, VA: USGS. http://waterdata.usgs.gov/nwis/ annual?site_no=02323500\&agency_cd=USGS\&p or_02323500_7=2396494,00060,7,1931,2013\&ye ar_type $=W \&$ referred_module $=s w \&$ format $=$ rdb
Servicio Geológico de los Estados Unidos (USGS). 2014c. Surface-Water Annual Statistics for Florida. USGS 02366500 Choctawhatchee River near Bruce, Florida. Reston, VA: USGS. http://waterdata.usgs.gov/ $\mathrm{fl} /$ nwis/annual?site_no $=02366500$ \&agency_cd=USG S\&por_02366500_2=2396875,00060,2,1931,2014\&ye ar_type $=$ W\&referred_module $=s w \&$ format $=$ rdb

Servicio Geológico de los Estados Unidos (USGS). 2014d. Surface-Water Annual Statistics for Florida. 02376033 Escambia River near Molino, Florida. Reston, VA: USGS. http://waterdata.usgs.gov/ $\mathrm{fl} /$ nwis/annual?site_no $=02376033$ \&agency_cd=USG S\&por_02376033_1=2396992,00060,1,1984,2014\&ye ar_type $=W \&$ referred_module $=$ sw\&format $=r d b$

Servicio Geológico de los Estados Unidos (USGS). 2014e. Surface-Water Annual Statistics for Florida. USGS 02246500 St. Johns River at Jacksonville, Florida. Reston, VA: USGS. http://waterdata.usgs. gov/fl/nwis/dv?cb_00060=on\&format=rdb\&site_ no $=02246500 \&$ referred_module $=$ sw $\&$ period $=\&$ begin date $=1971-10-01 \&$ end_date $=2014-11-02$

Servicio Geológico de los Estados Unidos (USGS). 2007. EDNA Derived Watersheds for Major Named Rivers. KML Watershed Index. Reston, VA: USGS. http://edna.usgs.gov/ watersheds/kml_index.htm

Instituto de Alimentos y Ciencias Agrícolas, Universidad de la Florida, Centro de Educación de Temas Públicos (UF/IFAS PIE Center). 2016. Water Quantity \& Quality. Gainesville, FL: UF/IFAS PIE Center. http://www.piecenter. com/issues/water/water/ 